\title{
Anne-Marie et Pierre Petrequin, avec la collaboration d'Olivier Weller, Objets de pouvoir en Nouvelle-Guinée. Approche ethno-archéologique d'un système de signes sociaux
}

\section{Gilles Bounoure}

\section{OpenEdition}

\section{Journals}

Édition électronique

URL : http://journals.openedition.org/jso/859

DOI : $10.4000 /$ jso.859

ISSN : 1760-7256

Éditeur

Société des océanistes

Édition imprimée

Date de publication : 1 juin 2007

Pagination : 153-155

ISBN : 978-2-85430-010-9

ISSN : 0300-953x

\section{Référence électronique}

Gilles Bounoure, «Anne-Marie et Pierre Petrequin, avec la collaboration d'Olivier Weller, Objets de pouvoir en Nouvelle-Guinée. Approche ethno-archéologique d'un système de signes sociaux », Journal de la Société des Océanistes [En ligne], 124 | Année 2007-1, mis en ligne le 23 avril 2008, consulté le 24 septembre 2020. URL : http://journals.openedition.org/jso/859 ; DOI : https://doi.org/10.4000/jso.859 
d'étranges charges contre les ethnologues néo-calédoniens de jadis, et spécialement Maurice Leenhardt pour sa «notion de personne en Mélanésie » (pp. 77-81, avec moins de virulence toutefois qu'Alban Bensa qui dénonce l'« ahurissante conviction du missionnaire », p. 13). Dans les bouleversements de toutes sortes auxquels sont aujourd'hui soumises les sociétés traditionnelles du Pacifique, même les meilleures observations ethnologiques d'autrefois perdent forcément de leur validité, mais leur en faire grief relève de cette faute de méthode que l'historien espagnol Salvador de Madariaga a nommée «l'anachronisme psychologique ». Depuis plus d'un quart de siècle, les ethnologues principalement d'expression anglosaxonne qui travaillent dans le reste de la Mélanésie « transculturelle » se concentrent de plus en plus sur la psychologie individuelle et la notion de « personne », sous l'influence notamment de Do Kamo (première édition en anglais, Chicago, 1979 et commentaire de James Clifford, Person and Myth, en 1982), comme l'ont souligné d'excellents chercheurs (A. L. Epstein, Michele Stephen, etc.) certainement aussi scrupuleux que dépourvus de toute sympathie pour les missionnaires. Il est dommage que Yoram Mouchenik se soit privé de leurs apports ou de leurs débats qui intéressaient directement sa recherche (tels ceux suscités par l'article de Schwartz, "Cult and Context : the Paranoïd Ethos in Melanesia », Ethos 1, 1973, évoqués par A. L. Epstein dans le JSO 110, 2000, à comparer avec le contenu allusif et vague de la note 217 p. 174 de L'enfant vulnérable sur la sorcellerie et « l'aspect paranoïaque des relations sociales » qu'elle induirait). Il est difficile de se prévaloir de la « rencontre des disciplines » en laissant de côté une proportion aussi importante, en volume comme en portée, des récentes recherches internationales et «transculturelles» dans l'ensemble de la Mélanésie.

On n'aurait garde d'oublier que l'auteur, psychothérapeute, cherchait avant tout à alléger la détresse de ses patients et de leur entourage, et la générosité de son engagement, ici comme sur d'autres terrains d'urgence (comme le rappelle Marie-Rose Moro), force la sympathie. La situation actuelle de la psychothérapie et des aides à l'enfance en France « métropolitaine » suggère l'ampleur de leur pénurie présente, comme celle de leur misère passée, dans les îles Loyauté. L'auteur a évidemment raison de dénoncer là des vestiges du « temps des colonies », au risque d'indignations parfois mal ciblées. Yoram Mouchenik ne dissimule pas ses difficultés au milieu d'intervenants multiples, ne masque pas ses échecs, et présente prudemment sa démarche de « psychothérapeutique transculturelle » (ou complémentariste) comme principalement « heuristique », empirique, à l'épreuve des faits. L'impression ambiguë que laisse son livre tient à la façon qu'a l'auteur de s'appuyer sur le savoir ethnologique à des fins thérapeutiques tout en adressant plus ou moins explicitement le reproche aux ethnologues de jadis ou d'aujourd'hui de n'être pas logés à la même enseigne, celle de l'efficacité immédiatement constatable, dont les « juges de paix » sont la rémission ou la guérison des détresses psychiques. Mal fondé en raison, et encore moins en logique, ce reproche est désastreux dans ses effets.
Il semble admis aujourd'hui que psychothérapeutes et ethnologues institutionnels, en l'attente d'une éventuelle mise au pas de ces deux professions par un pouvoir qui les trouverait à la fois trop critiques et insuffisamment « rentables », sont davantage au service du public qu'ils envisagent que des institutions qui les paient, et la plupart de tous ces professionnels sont probablement alarmés non seulement par la «vulnérabilité » d'une partie ou d'une autre de ce public, mais par les signes multipliés d'un état d'urgence toujours croissant, qui met désormais en jeu la survie de milliards d'humains, imposant de mettre à la disposition de tous les outils ou les " armes » nécessaires au salut commun. Cette mise en commun, ou si l'on veut, cette " rencontre des disciplines", ne peut être que critique, et si elle a tout à craindre d'un irénisme plus ou moins « missionnaire », elle n'a rien à espérer des polémiques creuses relayées par ce livre, où les poses partisanes font d'autant plus déplorer l'absence d'analyses réellement militantes, globales et utiles à tous.

Gilles BOUNOURE

Anne-Marie et Pierre PÉTREQUIN, avec la collaboration d'Olivier Weller, 2006. Objets de pouvoir en Nouvelle-Guinée. Approche ethno-archéologique d'un système de signes sociaux, catalogue de la donation Anne-Marie et Pierre Pétrequin, préface de Patrick Périn (directeur du musée d'Archéologie nationale de Saint-Germain-en-Laye, MAN-RMN-CTHS, 552 p., environ 600 illustrations et cartes en noir et blanc et couleur, index, bibliographie.

Que le différend, ou même le conflit, entre les tenants de l'appréciation "esthétique» des objets venus d'Océanie (ou d'autres " civilisations extraeuropéennes ») et ceux de leur étude " anthropologique » soit loin d'être apaisé, ceux qui ont assisté successivement, dans les derniers jours de juin 2006, à l'inauguration du musée du quai Branly et à la présentation de la donation Pétrequin au musée de SaintGermain-en-Laye en ont reçu une nouvelle preuve de fait : l'organisation de ces manifestations en deux lieux distincts suffisait à ridiculiser les prétentions toutes « verbales » des responsables de la « mission de préfiguration » de la première institution à « dépasser définitivement cette vieille querelle », qui est née avec les musées et durera autant qu'eux. Les visiteurs ne manquaient pas d'autres points de comparaison entre le premier « événement » organisé en forçant les feux, les volontés et les budgets et le second, lui aussi préparé de longue date, lui aussi inscrit au registre des « priorités » nationales depuis des années, mais sans cesse retardé faute de fonds pour accueillir dignement cette donation. Quant aux publications, aucune de celles éditées, par brassées, à l'occasion de la première de ces manifestations ne s'approchait de près ou de loin de cet ouvrage scientifique considérable dont l'exposition de Saint-Germain était aussi destinée à marquer la parution.

Il serait injuste, à propos de cet ouvrage, de ne rien dire de l'exposition ni de la simplicité, de la chaleur et même de l'enthousiasme avec lesquels Anne-Marie et 
Pierre Pétrequin aussi bien que Patrick Périn, le directeur du MAN, accueillirent leurs visiteurs d'un soir. Parcourir la présentation des trois cents pièces sélectionnées pour cette exposition en suivant les explications d'Anne-Marie et Pierre Pétrequin leur fit mesurer de façon exceptionnelle (car de vive voix et de quelles voix vives !) que nombre d'objets d'apparence modeste, jugés indignes d'autres musées, revêtent, pour qui sait, une importance scientifique égale à celle de bien des « chefs-d'œuvre ». La ferveur communicative de leurs hôtes incitait même les habituels parasites des vernissages à délaisser leurs papotages pour regarder enfin de près des objets traditionnels océaniens et s'intéresser à leur distribution dans les vitrines. Quant à Patrick Périn, qui justifiait auprès des journalistes (comme dans sa préface au catalogue) l'entrée au musée d'Archéologie nationale de ces mille cinq cents objets de Nouvelle-Guinée en tant que matériel supplémentaire pour la « salle de comparaison » prévue dès la création de ce musée en 1865 et très largement étendue sous la direction d'Henri Hubert, il s'amusait visiblement qu'on lui objecte que, suivant le principe de la donation Pétrequin, sa " salle de comparaison » était appelée à absorber toutes les collections du musée du quai Branly, sinon même beaucoup de celles du Louvre.

Anne-Marie et Pierre Pétrequin avaient rappelé dans un précédent ouvrage (Écologie d'un outil : la hache de pierre en Irian Jaya (Indonésie), 2000 : 397) qu'ils en étaient venus à étudier les techniques traditionnelles encore observables aujourd'hui en Nouvelle-Guinée en tant que « préhistoriens et dans le but explicite de donner aux préhistoriens d'autres hypothèses à tester sur le passé que les seules traditions conceptuelles du monde archéologique », prenant aussitôt pour exemple « deux aires de production de lames polies en Europe » (Vosges du Sud, Alpes) semblant vérifier certains «aspects du modèle néoguinéen, où se mêlent d'un côté les déterminismes matériels et techniques et de l'autre les résultantes des fonctionnements sociaux, sans qu'il soit possible de faire l'économie des uns ou des autres pour appréhender la complexité des phénomènes ». Ces derniers mots paraissent résumer au plus près la démarche constante de ces deux préhistoriens « comparatistes », en même temps que l'ambition principale de ce dernier et monumental ouvrage. Objets de pouvoir en Nouvelle-Guinée intéresse évidemment aussi bien les océanistes que les préhistoriens. Quel bénéfice en tireront les seconds, il leur reviendra de l'apprécier. Quant aux premiers, ils se réjouiront d'abord de voir rassemblés, et généralement remaniés, non seulement des travaux qui avaient été publiés à leur attention (notamment dans ces colonnes, JSO 91, 1990-2 et 108, 1999-1 ou dans les «mélanges Gerd Koch », Gestern und Heute, 1998), mais aussi des articles donnés à des publications plus lointaines, telle la très remarquable enquête sur les flèches des Dani, parue en 1990 dans le Bulletin de la Société préhistorique française.

Ce qu'on ne tardera pas à nommer par commodité le « catalogue Pétrequin » défie le compte rendu « critique » du fait non seulement de son volume, de la complexité et du nombre des sujets abordés, mais aussi de l'expérience des auteurs, sans équivalent actuel ni « concurrence» pouvant autoriser d'autres chercheurs à des discussions d'ensemble, si bien qu'il faut s'en tenir ici à de brèves observations. La NouvelleGuinée concernée est surtout l'actuelle partie indonésienne, ses Hautes Terres et la région du lac Sentani où les auteurs ont fait de nombreux et fructueux séjours, mais avec des explorations ou des incursions s'étendant aux rivages de la mer d'Arafura au sud, à l'ensemble du bassin du Sépik à l'est et aux Moluques au nord : le «terrain » choisi (et présenté de façon très vivante dans une longue introduction) est le lieu de rencontres entre " civilisations " anciennes probablement le plus riche qu'offre toute l'Océanie, et les auteurs apportent souvent des éléments nouveaux sur ces rencontres. Sur le plan de la culture matérielle, que les préhistoriens sont particulièrement entraînés à étudier dans le moindre détail, l'ouvrage envisage successivement les objets de parure ou de parade ( Arcs, filets et perles : la mise en scène individuelle », pp. 52173), la production, le commerce et l'usage de minéraux de haut prix («Cuire le sel et cuire la pierre », pp. 174-318) et enfin ceux de la céramique («Des pots pour les échanges », pp. 320-526). Cette dernière partie constitue, pour la Nouvelle-Guinée, le premier exposé de quelque ampleur jamais consacré à ce sujet en langue française.

Attirés par tout ce qu'ont de spectaculaire et fascinant les sociétés étudiées (et abondamment photographiées dans le livre) en tant que vestiges contemporains des "âges lithiques ", mais aussi par le style remarquablement clair des auteurs et l'excellente organisation du volume (j'ai mis quelque temps à trouver la légende du spécimen 87913 reproduit p. 304, beau manche de battoir à sagou à tête pivotante de style Geelvink, décrit p. 39 sans renvoi à la p. 304, mais cet oubli semble unique), les lecteurs, qu'on peut ainsi espérer nombreux, y feront des découvertes souvent comparables à celles que leur avait procurées Pamela Swadling dans Plumes from Paradise (1996), le premier ouvrage à révéler à un assez large public les acquis et les ressources de l'archéologie en Nouvelle-Guinée. Mais, rapportée à l'approche principalement historique de Pamela Swadling, la méthode d'Anne-Marie et Pierre Pétrequin est largement différente, et emprunte fréquemment le chemin inverse, consistant à partir du présent pour remonter dans le passé. Par la diversité de ses champs d'étude plus ou moins étroitement corrélés, ce qu'ils entendent par "ethno-archéologie » se rapproche aussi largement du projet d'ethnologie " globale » mené en 1974 par Gerd Koch et son équipe berlinoise chez les Eipo. L'enquête déjà citée sur les flèches de chasse et les flèches de guerre des Dani, mettant en évidence une composition des « paquets de flèches » correspondant à chaque tranche d'âge ayant des domaines d'activité privilégiés, est un modèle, non pas seulement de l'excellente méthode des auteurs, mais de recherche débouchant sur des apports immédiats et plus lointains, eu égard par exemple aux dizaines de milliers de flèches néo-guinéennes aujourd'hui entreposées à peuprès indistinctement dans les réserves des musées, au grand embarras de leurs conservateurs. 
À défaut de pouvoir signaler tous les résultats factuels de cette recherche monumentale, on s'arrêtera pour finir à cette autre ambition centrale du volume, étudier la culture matérielle sous l'angle d'un «système de signes sociaux » c'est-à-dire, si l'on ose emprunter ces termes à Maurice Godelier, déterminer la part de « l'idéel» dans « le matériel». Que tous les produits de l'industrie humaine soient à un titre ou à un autre des « objets de pouvoir » ou « de prestige », c'est un lieu commun exploité de nos jours aussi bien par les marchands d'art primitif que par les vendeurs d'automobiles. Anne-Marie et Pierre Pétrequin concluent de leurs observations et de leurs collectes d'objets qu' «à travers tous ces signes matériels s'exprime le besoin de se différencier et d'exprimer son pouvoir sur les autres. » (p. 529). De leur séjour chez les Eipo, Gerd Koch (Malingdam, 1984) et son équipe avaient au contraire conclu à l'absence de tout « pouvoir sur les autres » dans cette société « acratique » ou « anarchique » composée d'êtres parfaitement accomplis sur le plan individuel, mais il est vrai encore préservée de toute influence extérieure. Comment est né ou s'est imposé ce «besoin» de "pouvoir sur les autres »- supposé que cette dernière expression ait partout le même sens, en Nouvelle-Guinée comme en Occident -, c'est un vaste pan de leurs préoccupations que les océanistes ethnologues disputeront sans doute à leurs collègues préhistoriens qui s'y aventurent à la faveur de leur " approche ethnoarchéologique ». On peut ainsi s'attendre à ce que le «catalogue Pétrequin » donne rapidement lieu à des développements de premier intérêt, soit dans l'exploitation du colossal matériel historique et archéologique qu'il livre aux chercheurs, soit dans l'appréciation des hypothèses ethnologiques qu'il présente à la discussion.

\section{Gilles BOUNOURE}

Hermione Waterfield and J. H. C. KIng, 2006. Provenance. Twelve Collectors of Ethnographic Art in England, 1760-1990, Somogy-Musée BarbierMueller ${ }^{5}$, Paris-Genève, 176 p., bibliographies en fin de chapitre, index, très nombreuses ill. noir et blanc et couleur.

Quelque jugement qu'appelle ce petit chapitre de l'histoire « esthétique » et coloniale de l'Europe, c'est indiscutablement à l'initiative de particuliers, collectionneurs ou marchands, que les spécimens ethnographiques y ont été promus au statut d'objets d'art « primitif », et de mieux en mieux étudiés, mis en valeur ou même « en beauté » à proportion que leur cote augmentait et qu'ils suscitaient peu à peu la curiosité du grand public et des spéculateurs, notamment à raison des prix élevés qu'ils commençaient à atteindre. Entre autres effets multiples, cette évolution du " goût », à laquelle Paris doit son tout récent musée, s'est accompagnée d'un intérêt ambigu pour le cheminement de ces objets une fois parvenus en la possession d'Occidentaux, intérêt que les « cartels » de ce même musée sont loin de décourager («Ancienne collection Paul Guillaume », " Ancienne collection Charles Ratton ». etc.). Ce livre, dont le titre reprend l'intitulé d'une rubrique désormais classique dans les notices descriptives des objets « primitifs » présentés en vente publique, rassemblant ce qu'on peut dire de leur provenance européenne (« pedigree » dans le jargon des spécialistes !), offre une contribution importante à l'histoire de la circulation des objets anciens d'Afrique, d'Océanie et d'Amérique dans un pays qui fut longtemps la principale «plaque tournante » de ce secteur du marché de l'art.

En premier lieu, les Britanniques avaient accumulé un volume de spécimens ethnographiques sans équivalent dans le reste de l'Europe, du fait de l'extension et de la longévité de leur empire colonial, spécialement dans le Pacifique, avec toutes les allées et venues induites par sa mise en place et son maintien, de missionnaires, marins, fonctionnaires, commerçants ou aventuriers... Ces objets pour la plupart en mains privées ont longtemps constitué une partie du fonds de commerce des brocanteurs et autres marchands de curiosités en tout genre. Mais, comme le remarque Jeremy MacClancy («A Natural Curiosity : The British market in primitive art », Res, 15, 1988, repris dans J. MacClancy ed., Contesting Art. Art, Politics and Identity in the Modern World, Oxford, 1997, article non cité par H. Waterfield parce qu'il sort des sujets qu'elle envisage, mais dont les analyses complètent utilement son livre), pendant tout une partie $\mathrm{du} \mathrm{Xx}^{\mathrm{e}}$ siècle, « British social anthropologists ignored material culture and ethnologists were interested only on taxonomy ». Ces derniers, s'ils étaient en charge de musées, pouvaient ainsi être amenés à échanger des « doublons » contre des pièces susceptibles de «compléter» leurs collections, qui n'intéressaient du reste qu'une petite « coterie of dealers, collectors and curators » (MacClancy, ibid.). Tel est le petit monde qu'étudie et éclaire ce livre.

Longtemps directrice du département des arts africains, océaniens et américains de Christie's, Hermione Waterfield y a développé non seulement une expertise exceptionnelle à la fois d'ampleur et d'acuité, entre des aires d'expressions artistiques si variées, mais une connaissance à la fois diachronique et synchronique du « milieu » international de l'art primitif excédant très largement ce qu'exige le fonctionnement d'une maison de ventes publiques, serait-elle la première au monde. Dans ses descriptions d'objets, elle s'assura aussi pendant de nombreuses années le concours du grand africaniste William Fagg, ancien keeper du département ethnographique au Bristish Museum, et l'un des premiers muséologues britanniques à s'intéresser à la valeur proprement artistique des spécimens ethnographiques. On ne peut être surpris que pour l'introduction et deux chapitres de ce livre, elle se soit ménagé la collaboration d'un autre ancien conservateur du B. M., prestigieux lui aussi, Jonathan C. H. King, auteur de travaux de référence sur l'art des Indiens d'Amérique du Nord. Réputées immorales en France, de telles synergies entre experts venus du secteur public et du monde marchand n'ont rien que 